\title{
Composite Materials Flaws Detection and Measurement by Infrared Thermography
}

\author{
By A. Mihai*, F. Stefanescu*, A. Dumitrache-Rujinski* and G. Neagu* \\ * Univ. POLITEHNICA of Bucharest, Romania, sandamihai@yahoo.com
}

\begin{abstract}
Composite materials are large used today in various industrial fields such as: aeronautics, military equipment, pipelines for oil transport; frequently must meet serious requirements about the quality. This paper presents the results of an experimental study on the possibilities and limits of flaws detection and measurement in composite materials by infrared thermography. In a research contract developed in our university were designed and processed samples with artificial defects in composite materials: CFRP, GRFP and Dyneema. The main aim of experimental research was to assess size of flaws positioned at different depths related to the examination area. The samples were investigated by infrared thermography and then the results were verified by different ultrasonic methods.
\end{abstract}

\section{Introduction}

Infrared thermography (IRT) represents a valid tool already known to detect defects of different composite materials, mainly due to some particular advantages, related to other non-destructive testing methods [1.2]. One advantage is that thermography offers inspection results, practically, instantaneously. Secondly, this method is contactless, thus allowing rapid in-situ inspection of large areas. Thermography is also environmentally safe. Today, the defect detection is not enough. It is often necessary to measure the defects dimensions [3]. A large experimental research has been developed to establish the minimum detectable defect size for different composite materials and position of defect in depth. Variability of results and measurement uncertainty often causes the discouragement of operators to estimate the size of defects by IRT inspection. To measure the defect dimensions is a subject of many actual studies $[4,5,6]$. Although, infrared thermography is limited to the detection of relatively shallow defects (a few millimeters under the surface), however, the most common defects of composite materials widely used in the aircraft, automotive, oil industries etc. such as delaminations, disbonds, impact damages, can be effectively detected and sometimes quantified using active thermographic techniques. The main limits of infrared thermography are: the difficult interpretation of images due to the surface reflections, heat loss by convection or absorption of radiation by atmospheric components and the low detectability of the defects located deeper than a few millimetres of the examined surface. To measure the defect dimensions is a subject of many actual studies [7], [8], [9].

Our research team has approached the examination of polymer composite materials by combining two methods: firstly, a method which does not require direct contact between the material examined and the examination system components - infrared thermography - and a second method, a local one, requiring contact, based on ultrasounds (UT) to confirm the results obtained by IRT. The research goal was to estimate the uncertainties of the infrared thermography measurements, taking into account the main advantages of this method: efficiency of examination for products with large surface areas.

\section{Theoretical aspects}

According to the Fourier's law, the conductive heating rate is the rate of heat flow: under steady state conditions it is directly proportional with the thermal conductivity of the object, cross sectional area of the object, through the heat flows, and the temperature difference between the two ends of the object, and inversely proportional with the length or thickness of the object. For heat flow to continue at the same rate it is necessary to introduce energy in the system, so that the temperature difference will not start to decrease. In the steady state the heating power is equal to the cooling power and in the transitory conditions, the temperature changes over time.

In order to solve the Fourier's differential equation of the thermal conduction in a particularly situation of transient conditions, it is necessary to know the real conditions of process development which determine the univocity of the process: geometrical conditions, which describe the dimensions and shape of the body; physical conditions, which include the material thermal properties; initial conditions, which establish the body temperature in the initial moment; boundary conditions of first type, which establish the temperature distribution at the body surface related to time; boundary conditions of the second type, which refer to the time variation of the thermal flux density at the heat exchange surface; and boundary conditions of the third type, which establish the manner of heat exchange between the solid material surface and the environment.

The theoretical study allows establishing the examination parameters $[4,5]$. The heat transfer by conduction is essential in differentiating the zones with defects found inside the material. The modelling of phenomena which are developed during the thermographic processes must lead to the choice of some criteria to establish the main parameters 
of examination (heating thermal flux, heated surface temperature, optimal time of recording etc.), such as to assure the detection of all flaws with the size bigger than the maximum acceptable flaws. We have used an electrical modelling to study the influence of the main factors on the temperature contrast, so that the optimal parameters to be established. Figure 1, shown variation of the thermal contrast $\left(T_{d}-T\right)$ as a function of flaw depth position and the registration time. The results leaded to a family of curves for different times of thermal image recording, in the cooling process.

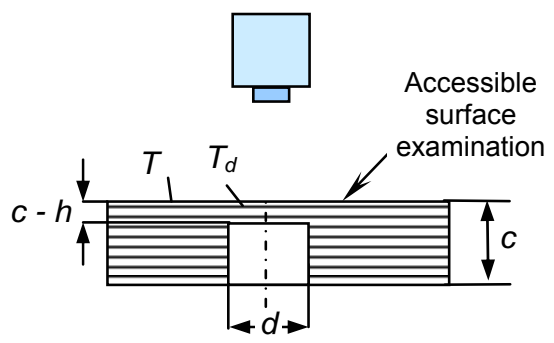

$c$ - material thickness;

$d$ - characteristic dimension of the defect; $c-h$ - distance from the accessible surface examination and the defect depth position; $T$ - temperature on the surface without defect; $T_{d}$ - temperature on the surface with defect.

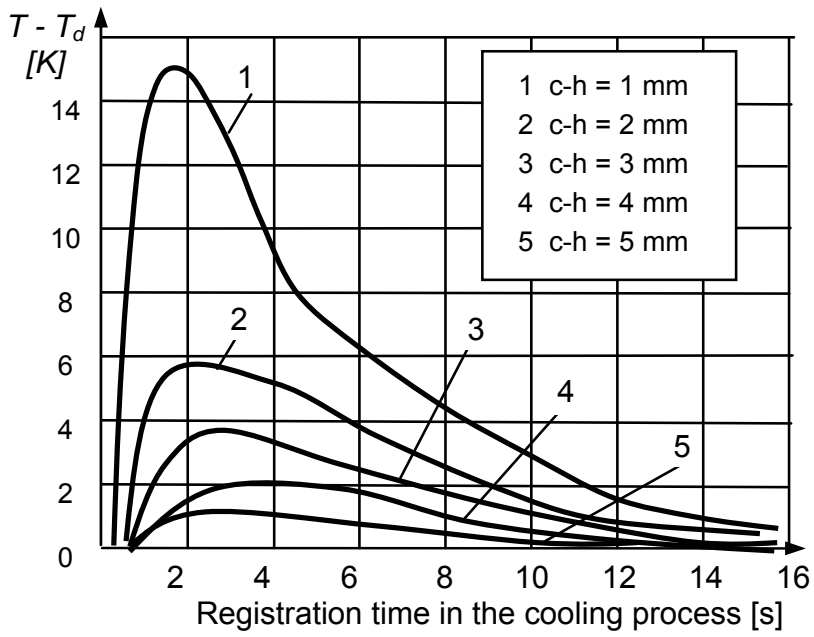

Fig. 1. Thermal contrast as a function of the flaw position and the registration time.

\section{Samples and experimental setup}

Samples were designed and processed from different composite materials, such as: polymeric reinforced with carbon fibres, CFRP, polymeric reinforced with glass fibres GFRP, and Dyneema - high performance polyethylene fibre (HPPE). The samples size was:

- $\quad a \times b \times c: 100 \times 100 \times 10,80 \times 80 \times 10,70 \times 80 \times 10 \mathrm{~mm}$;

- $\quad$ artificial defects - many flat bottom holes;

- diameter of holes, $\mathrm{d}=3,4,5,6,7,8,9,10,12$ and $14 \mathrm{~mm}$;

- depth of holes, $\mathrm{h}=2,3,4,5,6,7,8$ and $9 \mathrm{~mm}$, and different placement (Figure 2).

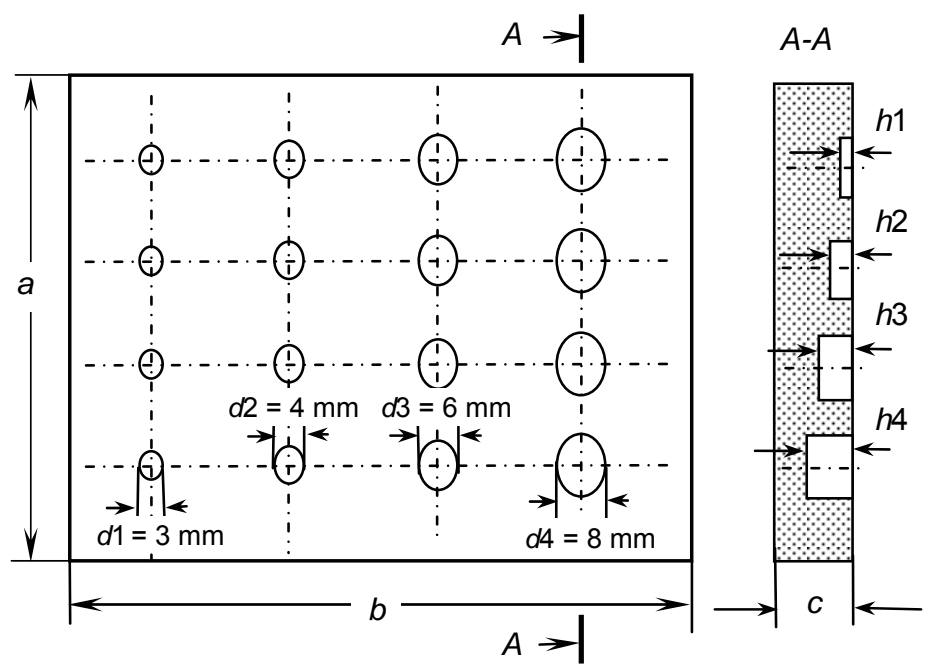

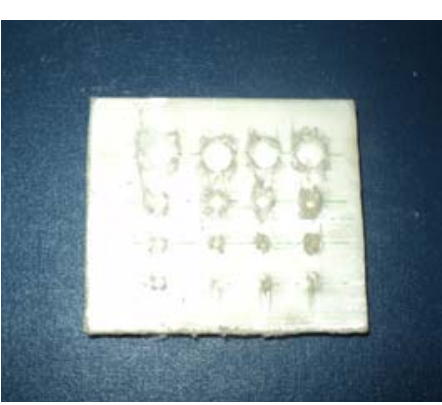

Dyneema sample

Fig. 2. Samples from different composite materials with artificial flaws and dimensions.

Figure 3 presents experimental setup used for the IRT inspection. The samples were investigated by infrared thermography, different techniques, mainly active thermography, with the aim to establish: the optimum scheme and parameters of examination, as well as measurement uncertainty of the size of defects thermographical detected. 
The equipment used was: IR Camera FLIR SC 640 Focal Plane Array (FPA) detector, uncooled microbol. $640 \mathrm{x}$ 480 pixels, spectral range: 7.5 to $13 \mu \mathrm{m}$; field of view/min focus distance $24^{\circ} \times 18^{\circ} / 0.3 \mathrm{~m}$, spatial resolution (IFOV) 0.65 mrad; thermal sensitivity $60 \mathrm{mK}$ at $30^{\circ} \mathrm{C}$, image frequency $30 \mathrm{~Hz}$ non-interlaced; focus automatic or manual, temperature range $-40^{\circ} \mathrm{C}$ to $+1,500^{\circ} \mathrm{C}$, in 3 ranges; up to $+2000^{\circ} \mathrm{C}$; emissivity correction: 0.01 to 1.0 or select from listings in predefined materials list.

Parameters of IRT inspection were:

- ambient temperature, $T_{a m b}=20^{\circ} \mathrm{C}$;

- emissivity, $\varepsilon=0.96$;

- distance, $d=1 \mathrm{~m}$;

- $\quad$ humidity, $w=50 \%$;

- surface heating at $40{ }^{\circ} \mathrm{C}$ with hot air or flash lamp;

- examination on the same surface;

- $\quad$ recording during the cooling process at every $2 \mathrm{~s}$.

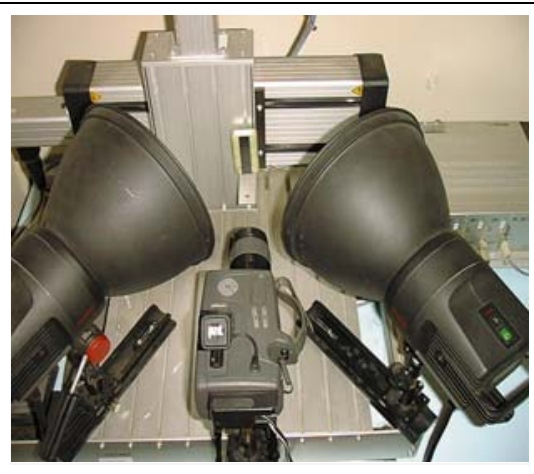

Active IRT Stand

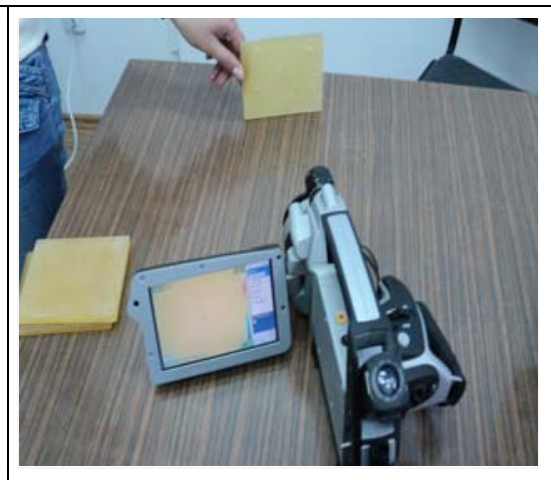

IR Camera

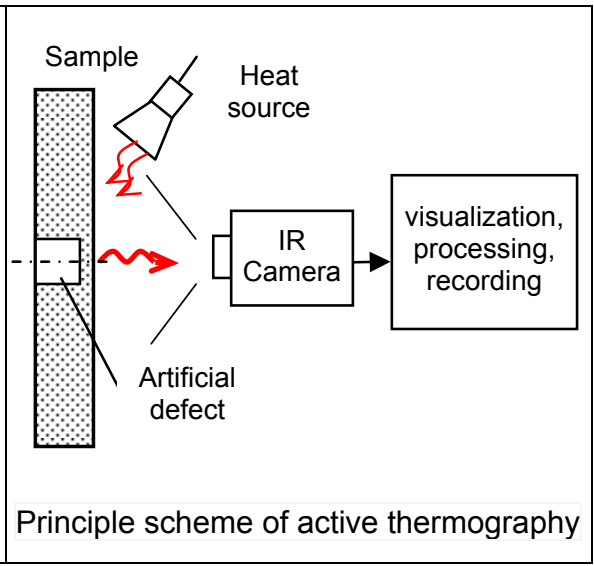

Fig. 3. Experimental setup used to investigate the samples with artificial defects.

To verify the IRT results, we have been applied ultrasound examinations. The samples were scanned with different transducers and frequencies and the results are A and C-scan images.

Figure 4 presents the experimental setup used to ultrasounds inspection.

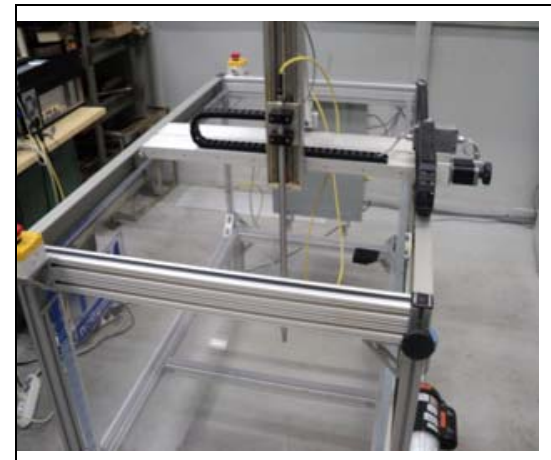

Ultrasound in immersion
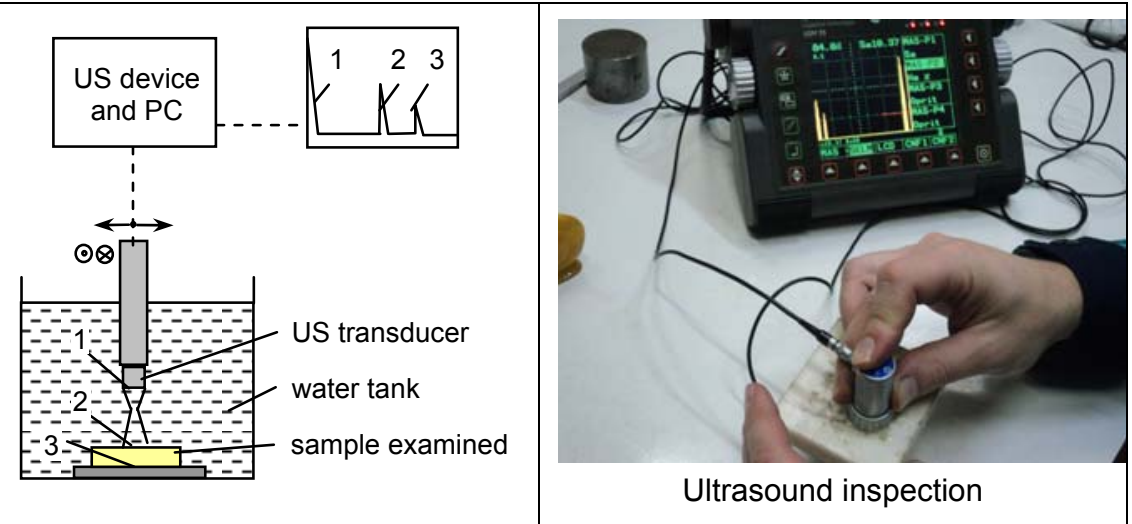

Fig. 4. Experimental setup used to investigate the samples with artificial defects.

Ultrasound systems were:

- $\quad$ NDT Automation - ultrasonic system to examine by immersion: frequency range $0,2-30 \mathrm{MHz}$, real time digitalization with $400 \mathrm{MHz}$ Increased inspection velocity and accuracy, TOF resolution 2,5 ns, high PRF of $20 \mathrm{kHz}$ Increased inspection velocity, amplifier 110dB dynamic range, DAC and TCG function;

- USM $35 \mathrm{XS}, \mathrm{GE}$, frequency ranges: 0.2 to $1 \mathrm{MHz} / 0.5$ to $4 \mathrm{MHz} / 0.8$ to $8 \mathrm{MHz} / 2$ to $20 \mathrm{MHZ}$, gain 0 to 110 $\mathrm{dB}$, measurement resolution: $0.01 \mathrm{~mm}$ within a range up to $99.99 \mathrm{~mm} / 0.1 \mathrm{~mm}$ within a range from 100 to $999.9 \mathrm{~mm} / 1 \mathrm{~mm}$ above $1,000 \mathrm{~mm}$, with evaluation in the frozen A-scan: $0.5 \%$ of the calibre range setting.

- $\quad$ Normal and focalised transducers: 1, 2, 4, 5, 8, 10, and $20 \mathrm{MHz}$. 


\section{Results}

The aims of research were to evaluate the level of performance in quantitative IRT inspection and to establish the level of uncertainties in the IRT measurements. After the samples design and processing, with different artificial flaws, the plan of experiments was accomplished and many IR thermograms of different samples and ultrasound $A$ and C-scan images were recorded and analysed.

Figure 5 presents some IRT images, as examples. The artificial defects can be clearly "seen" if the thermal, geometrical and the temporal parameters are adequate chosen and the registration is done at the right time, when the contrast is maximal.

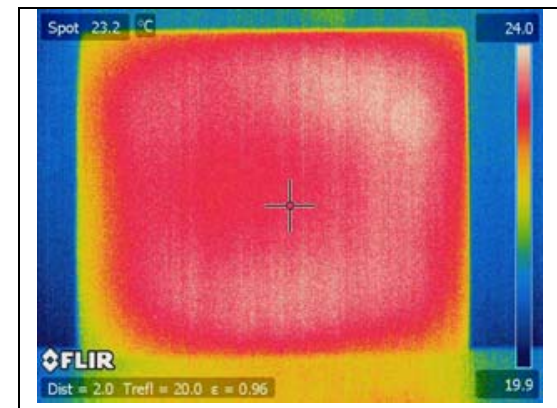

a

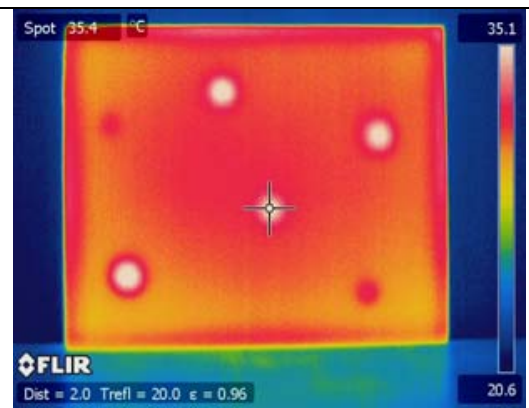

$b$

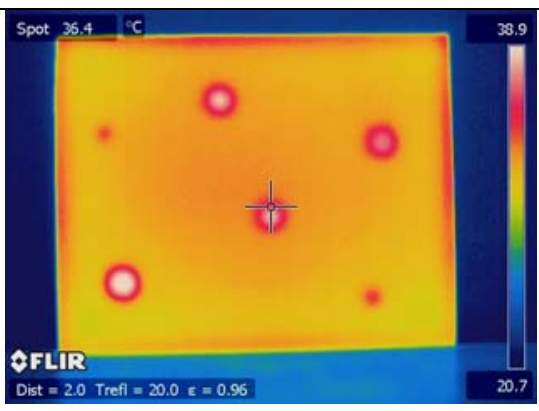

C

Thermal images of the samples of GFRP: $a$ - without defects; $b$ - with artificial defects (flat bottom holes located at 1 $\mathrm{mm}$ depth, diameters: 3,4 and $6 \mathrm{~mm}$, recorded during the cooling process after $2 \mathrm{~s} ; c$ - the same as $b$, recorded during the cooling process after $4 \mathrm{~s}$.

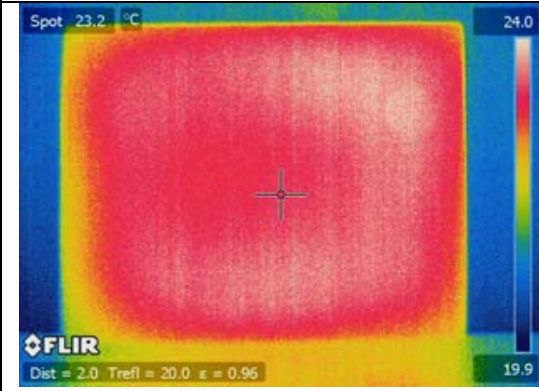

a

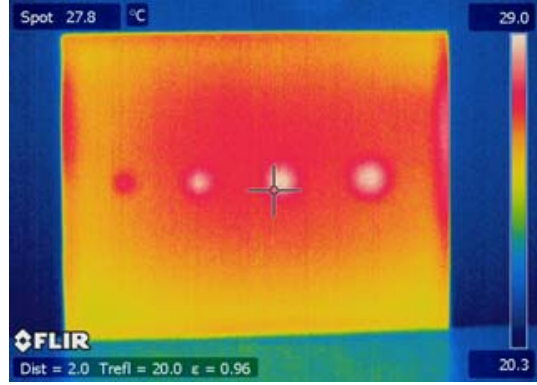

$b$

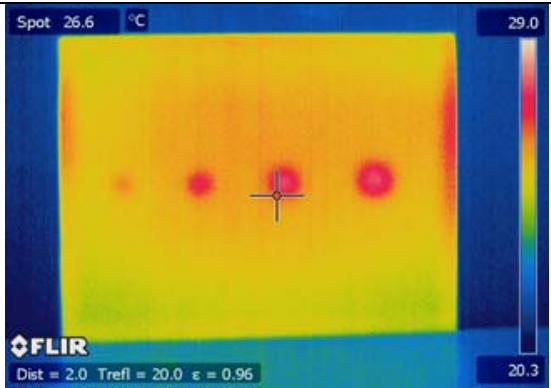

C

Thermal images of the samples of CFRP: $a$ - without defects; $b$ - with artificial defects (flat bottom holes located at 1 $\mathrm{mm}$ depth, diameters: 3, 4.5 and $6 \mathrm{~mm}$, recorded during the cooling process after $2 \mathrm{~s}$; $c$ - the same as $b$, recorded during the cooling process after $4 \mathrm{~s}$.

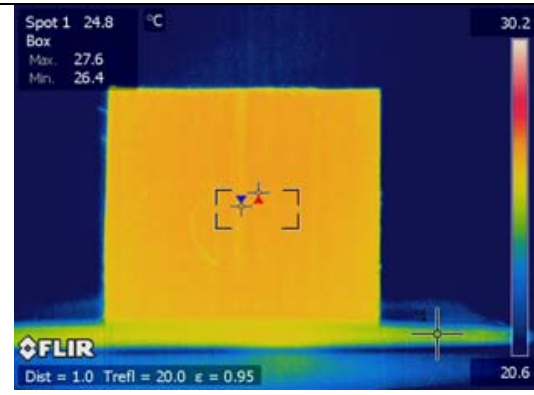

a

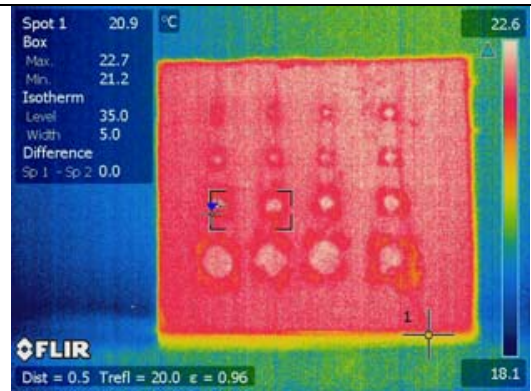

$b$

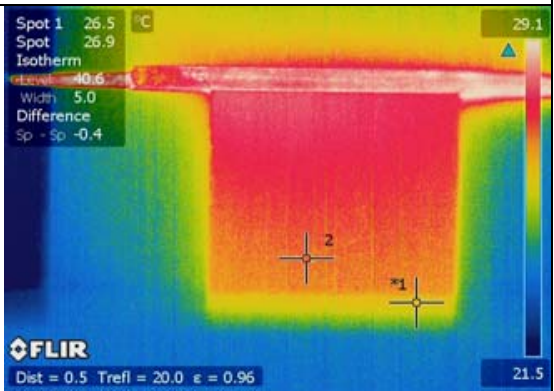

c

Thermal images of the samples of Dyneema: $a$ - without defects; $b$ - with artificial defects (flat bottom holes located different depth, diameters: 3, 4. 6 and $10 \mathrm{~mm}$; c-lateral, conductive heating.

Fig. 5. Thermal images of samples with artificial defects axially aligned and arbitrarily located.

The obtained thermograms revealed the good resolution and high level possibility to measure defects located near the surface accessible examination. Figure 6 shows, in a synthesized manner, the experimental results obtained by the IRT and US investigations. 

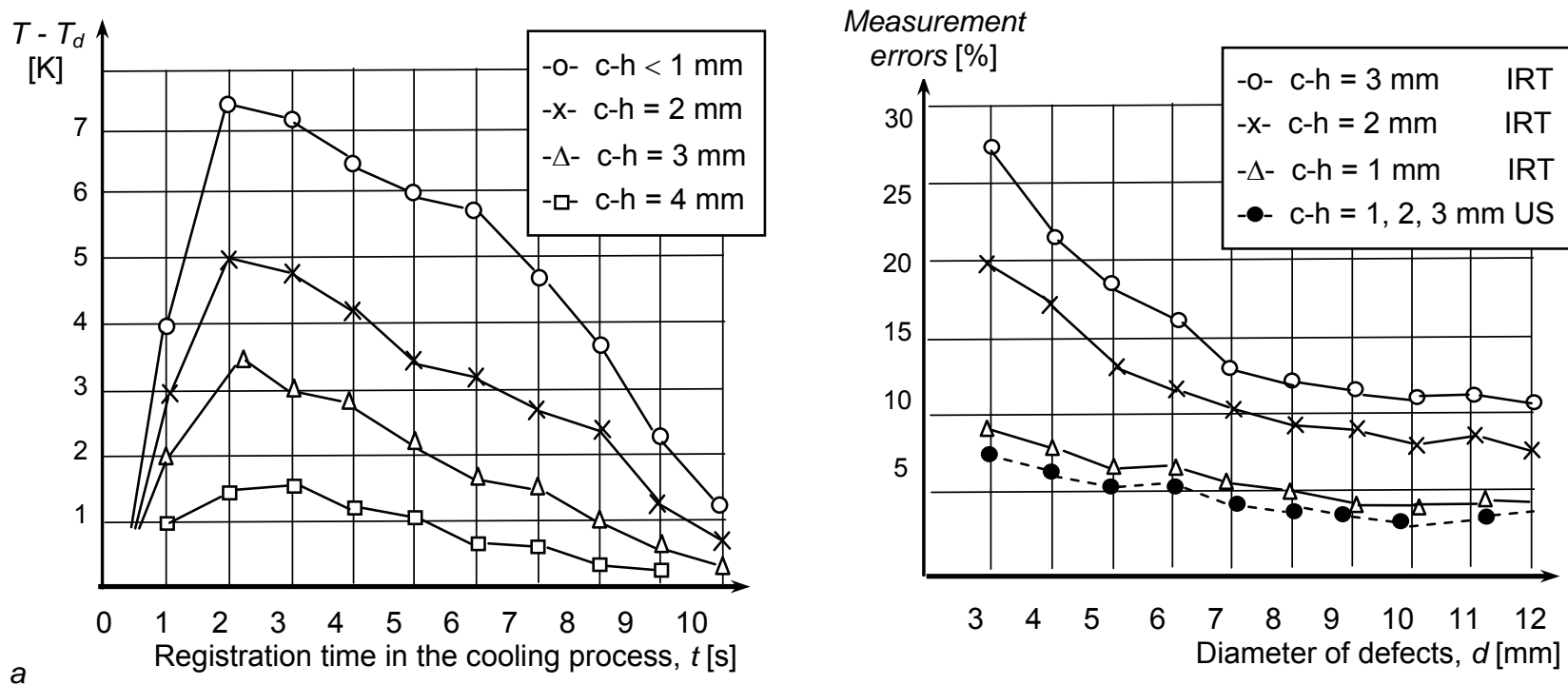

Fig. 6. Experimental results: a - thermal contrast variation depending on the position of the defect depth and image recording time; $b$ - variation of measurement accuracy depending on the diameter of the defect and its position in depth in IRT inspection, comparing with US results.

Figure 7 presents few ultrasound images, as examples.
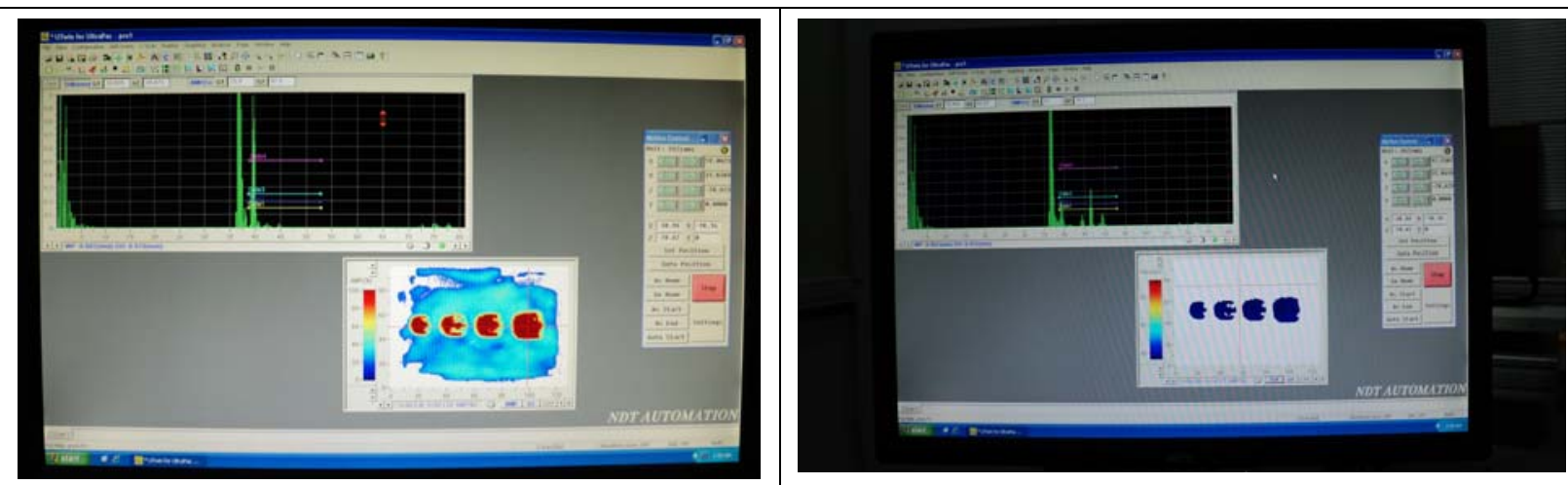

a

$b$
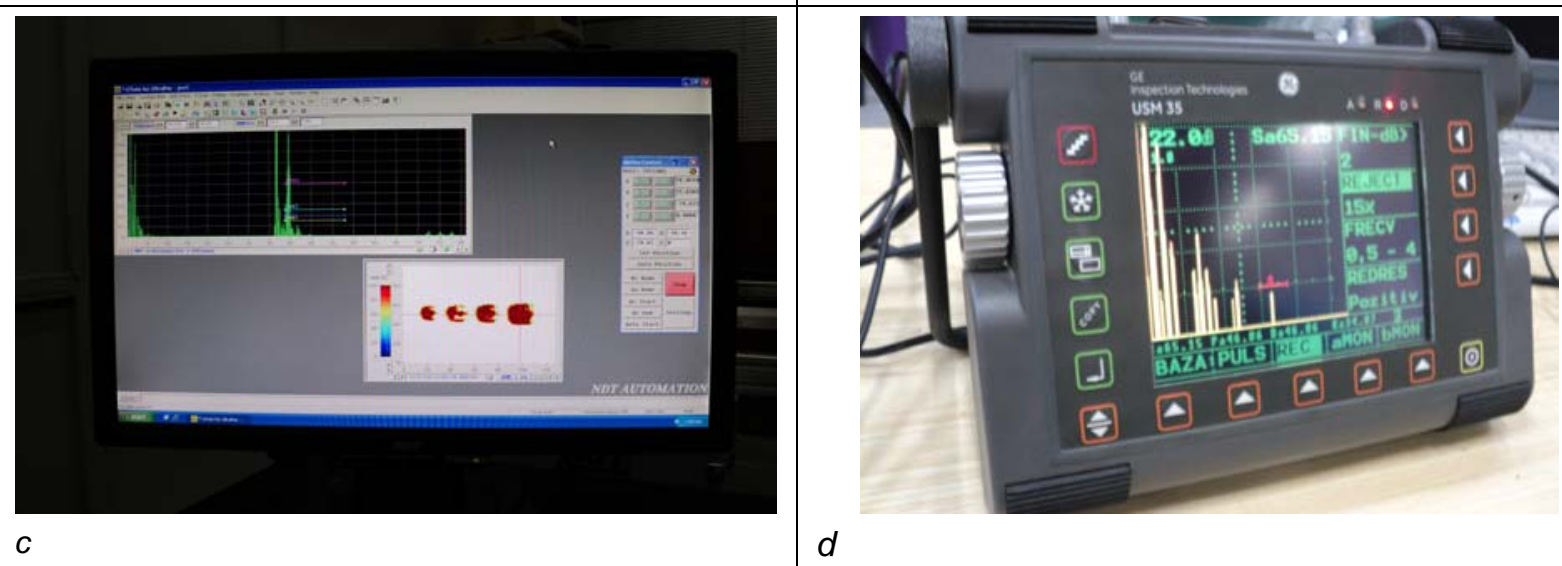

Fig. 7. Ultrasound images: results obtained by ultrasound inspection, both A-scan and C-scan images: a - CRFP - immersion examination, $f=5 \mathrm{MHz}, A$ and C-scan - results presentation, AMP (amplitude) function analysis; $b$ and $c-C R F P$ immersion examination, $f=5 \mathrm{MHz}, A$ and $C$-scan - results presentation, TOF (time of flight) function analysis; $d$-Dyneema - contact examination, A-scan results presentation, $f=8 \mathrm{MHz}$. 
The experiments showed the performances and limits of IRT inspection used as a measurement tool of the detected defects in comparison with different ultrasound methods.

\section{Comments and conclusions}

Generally, IR Thermography provides a very good resolution in the polymeric composite materials investigation, (CRFP, GRFP) except Dyneema material (this material is quite opaque to the ultrasonic and thermal waves).

In real terms, errors in thermographic inspection occur mainly of the following causes:

- incorrect assessment of emissivity, atmospheric temperature or ambient temperature, moisture, distance between camera and object;

- the influence of environmental radiation, detected by the IR camera directly and/or by reflections on the objects, located in the vicinity of the object examined;

- an incorrect assessment of atmospheric absorption and radiation phenomena;

- failures caused by the inaccuracy of the detector noise assessment.

Fortunately, in the quantitative non-destructive examination, the measurement of the temperature often is not required in absolute value. It is useful only to establish the temperature difference between two neighbouring areas: with defect and without defect. Low diffusivity and effusivity of polymers are advantages for detection and characterization of the defects. Thermography provides very good defect resolution. The materials CFRP, GFRP and other polymeric composite have a relatively high emissivity and therefore thermographic examination offers good results.

In the studied materials, the IRT measurements are comparable with the results obtained by ultrasounds investigation, when defects have diameter higher than $6-8 \mathrm{~mm}$ and depth of less than $1 \mathrm{~mm}$, In this domain, the value of the global uncertainty is quite similar, both in IRT and ultrasonic investigation.

For example, the level of uncertainty increases from $5 \%$ when the defect is located at a depth of $1 \mathrm{~mm}$ to $20 \%$ when the defect is located at a depth of $4 \mathrm{~mm}$ in a composite material reinforced with glass or carbon fibers, investigate by active IRT (flash heating).

However, the results are strongly affected by the surface features and external factors such as no uniform heating or environmental reflections. Advanced signal/image processing is required to reduce their impact. Thermographic inspection associated with ultrasonic examination provides an interesting means to evaluate the quality of these materials. Ultrasonic Inspection requires a long time to scan large surfaces with a step that depends on the geometry of the ultrasonic beam (hence the ultrasonic transducer geometry) in both ways: direct contact or immersion.

The researches conclude that the active infrared thermography is more suitable to rapidly detect the defect in large-area and the underwater ultrasonic C-scans are more suitable to quantitatively characterisation of the defect in local-area. The results of the experiments show that the active infrared thermography technique is a fast and effective inspection method for detecting the defects such as: delamination, disbond, impact damages and inclusions of the composites. The main limit regarding the IRT, using as non destructive method in quantitative inspection, is determined by the random reflections on the surface examined and because of thermal diffusion phenomenon that sometimes occurs quickly. However, in quality evaluation of polymeric composite materials with large surface, IRT inspection is irreplaceable. To diminish the uncertainty of the IRT measurements it is necessary to repeat investigation and/or to combine with other complementary methods, like ultrasounds inspection. The IRT inspection could offer good results in global examination and the associate methods could be used only local, or to confirm and validate the IRT results.

\section{Acknowledgment}

The main results are obtained in framework of the scientific national research contract, entitled: Composite structures resistant at dynamic loadings applied with high deformation speeds used in the field of collective protection HEAMIL, Contract-PCCA, nr. 209/2012.

\section{REFERENCES}

[1] Maldague X., „Theory and Practice of Infrared Technology for Nondestructive Testing”, John Wiley \& Sons, 2001.

[2] Maldague X., "Nondestructive evaluation of materials by infrared thermography", London Springer Verlag, 1993, new revised edition, John Willey\&Sons Pub. UK, 2001.

[3] Avdelidis N.P., Gan T-H., Ibarra-Castanedo C., Maldague X., „Infrared thermography as a non-destructive tool for materials characterisation and assessment" Thermosense, Thermal Infrared Appl. XXXIII, USA, 2011.

[4] Constantin N., Mihai A, a.a "Results of Nondestructive Inspection of Layered Composites using IR Thermography and Ultrasonics", Key Engineering Materials, Vols 413, p. 343-350, Trans. Tech Publication, Switzerland, 2009.

[5] Mihai A., Stefanescu F., Neagu G., "Quality Characterization of Composite Material Pipes by Thermographic Inspection", poster conf., MSE, Darmstadt, Germania, Aug. 24-26, 2010.

[6] Mihai, A., Constantin N., Stefanescu F., Neagu G., "Infrared Termography to Assess Impacted Pipes", WSEAS proceedings of the International Conference on Energy and Env. Tech, and Equipment p. 78-81, Buc. 2010. 